\title{
A Series of ILP models for the optimization of water distribution networks
}

\author{
NIKHIL HOODA $^{1, * \mathbb{D}}$, ASHUTOSH MAHAJAN $^{2}$ and OM DAMANI ${ }^{1}$ \\ ${ }^{1}$ Department of Computer Science and Engineering, Indian Institute of Technology, Bombay, Mumbai, India \\ ${ }^{2}$ Department of Industrial Engineering and Operations Research, Indian Institute of Technology, Bombay, \\ Mumbai, India \\ e-mail: hooda.nikhil@gmail.com
}

MS received 9 March 2019; revised 3 August 2019; accepted 1 September 2019

\begin{abstract}
The design of rural drinking water schemes consists of optimization of several network components like pipes, tanks, pumps and valves. The sizing and configuration of these network configurations need to be such that the water requirements are met while at the same time being cost efficient so as to be within government norms. We developed the JalTantra system to design such water distribution networks. The Integer Linear Program (ILP) model used in JalTantra and described in our previous work solved the problem optimally, but took a significant amount of time for larger networks-an hour for a network with 100 nodes. In this current work, we describe a series of three improvements of the model. We prove that these improvements result in tighter models, i.e. the set of points of linear relaxation is strictly smaller than the linear relaxation for the initial model. We test the series of three improved models along with the initial model over eight networks of various sizes and show a distinct improvement in performance. The 100-node network now takes only $49 \mathrm{~s}$ to solve. These changes have been implemented in JalTantra, resulting in a system that can solve the optimization of real world rural drinking water networks in a matter of seconds. The JalTantra system is free for use, and is available at https://www.cse.iitb.ac.in/jaltantra/.
\end{abstract}

Keywords. Water Distribution; Optimization; Integer Linear Program; Pipe Diameter Selection; Tank Configuration Selection.

\section{Introduction}

Piped water distribution networks are used to transport drinking water from common water sources to several demand areas. Therefore, the design of such networks is an important problem and has been studied in various forms over several decades.

A typical piped water distribution network, as shown in figure 1, consists of several infrastructure components like pipes, tanks, pumps and valves. The location and sizing of these components are determined as part of the network design. The network consists of one or more sources of water and several demand nodes. Each of these demand nodes is described by its elevation, demand and minimum pressure requirements. These nodes are connected by several links along which pipes have to be laid out to transport water from the source to each of the nodes. The network layout can be looped/cyclic (typically urban) or branched/ acyclic (typically rural). As the water flows through the pipes, the water pressure head reduces due to frictional losses. This loss, commonly referred to as headloss,

*For correspondence

Published online: 20 November 2019 depends on various factors like the diameter, roughness, flow and length of the pipe.

The pipe diameter selection problem consists of assigning pipe diameters to each link in the network. This selection is to be made from a discrete set of commercially available pipe diameters. Each link can be broken up into multiple segments, each consisting of pipes of different diameters or each link can be restricted to just one pipe diameter. In the most basic problem formulation, other components like tanks, pumps and valves are not considered.

Several approaches to the problem have been considered over the years. Early attempts at solving the pipe diameter optimization consisted of deterministic techniques. Linear Programming and its variants were some of the earliest attempts at the problem [1]. Dynamic programming [2] was also used to solve the network in stages rather than solving it entirely at once. This technique however faces difficulty in solving looped networks, since they are not amenable to being split into different stages. Since the problem consists of nonlinear equations, Non-Linear Programming (NLP) [3] techniques were applied as well. They however deal only with continuous diameters of pipes and thus require 


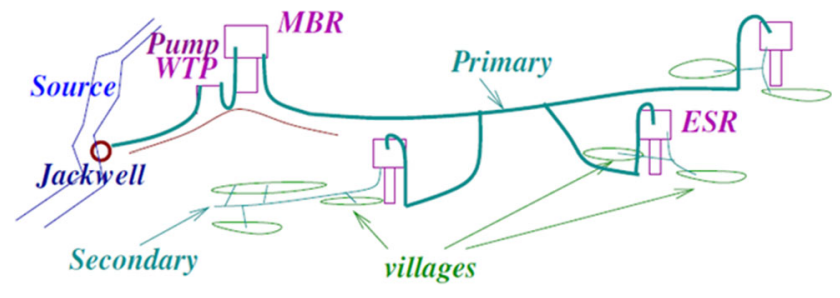

Figure 1. Components of a typical Rural Piped Water Scheme. Water is pumped from the source to the Water Treatment Plant (WTP) and then to the Mass Balancing Reservoir (MBR). The Primary Network then transports water from the MBR to the tanks/Elevated Storage Reservoirs (ESRs), and then finally the Secondary Network connects the tanks/ESRs to individual villages (courtesy: CTARA, IITB).

some form of rounding to determine diameters that are available only in discrete sizes. To take care of this discrete nature of pipes, Integer Linear Programming Techniques (ILP) [4] were used. The advantage of these techniques is their ability to provide non-ambiguous and consistent results. However, due to the NP nature of the problem [5], the computational demand rapidly increases with the size of the network. As a result, lot of work in the past couple of decades has focused on the use of meta-heuristic techniques. These techniques employ heuristics to traverse large search spaces and find near-optimal solutions. Several approaches like genetic programming [6, 7], tabu search [8], shuffled frogs [9], etc. fall in the wide umbrella of meta-heuristics. These techniques however do not guarantee optimal results since they do not cover the entire search space and are stochastic in nature, i.e., they may provide different results when repeated.

Networks, however, do not consist of pipes and nodes exclusively. Other components like tanks, pumps and valves are also part of any network design. Typically, rural water networks are gravity fed, i.e. water from the source flows downstream to the various demand nodes in the network. The water head along the links decreases gradually due to headloss. For certain nodes, there might not be enough water head in the system to ensure that their demands are satisfied. In such cases, pumps are used to provide additional head to the system. Depending on the network configuration, pumps can be installed at the source or at various points in the network, as per requirement. Though the use of pumps might decrease the cost of pipes (since pipes with smaller diameters would be required), and in some cases their use may be unavoidable, they cause a significant burden on the network operation since pumping requires electric supply. Therefore, apart from a one-time capital cost of infrastructure, there is now an additional operational cost of running the water network.

Conversely, in certain networks the source might be at a significantly higher elevation than the rest of the network. This would result in excess pressure throughout the network, which may cause pipes to burst. Therefore pipes with higher pressure rating would need to be installed, causing a significant increase in capital cost. In such cases, pressurereducing valves maybe employed to artificially reduce the excess pressure in the system. Valves may also be installed to restrict flow through certain pipes for maintenance and operational purposes.

Tanks help provide buffer capacity to the network. Since demand varies with time, tanks can be filled during low demand periods and provide water in times of higher demands. They can also be used to manage distribution of water and act as intermediary sources. This is particularly relevant in the case of areas where water is scarce. Tanks are filled from the source and they in turn act as secondary sources to the final demand nodes. The inflow and outflow of the tanks are managed to ensure equitable and timely distribution of water. In the absence of such a system, upstream nodes with high pressures will draw majority of the water from the source, leading to insufficient supply to downstream nodes.

Pumps were the earliest component to be considered during network optimization, in addition to the selection of pipe diameters, although they were restricted to a single pump at the source [10]. Tanks and valves were incorporated within meta-heuristic frameworks [6, 8, 9]. The networks considered in these studies are urban, where the role of tanks is to act as buffers to be used during periods of high demand. The choice to be made is the location, size and height of the tank. The number of tanks to be installed is fixed. But as mentioned earlier, water-scarce areas use tanks as secondary sources rather than buffers. The demand nodes are partitioned and allocated to individual tanks. The source supplies water to the tanks in a primary network. The tanks in turn supply water to their allocated nodes in secondary networks. The cost of the scheme can vary significantly depending upon the number of tanks and the partition of nodes to these tanks.

Indian government bodies use software like WaterGems [11] and BRANCH [12] to design water distribution networks [13-16]. They consist of only pipe diameter optimization. Other components, i.e. pumps, tanks and valves, are considered manually by the design engineer, relying on his/her experience and intuition [14]. We have implemented a network deign software, JalTantra, which includes tanks, pumps and valves in addition to the pipe diameter selection problem. JalTantra is a free-to-use webbased application available at https://www.cse.iitb.ac.in/jal tantra/. JalTantra has been officially adopted by the government of Maharashtra as a tool in the design process of their drinking water schemes.

In [17] we presented the first version of JalTantra, which included just the pipe diameter optimization for branched networks (typical in the case of rural areas). It used a Linear Programming model and thus solved the problem quickly and optimally. This allowed even networks of a thousand nodes to be solved in a couple of seconds. In [18], we extended the model to include tanks. The added 
complexity, of considering both primary and secondary networks simultaneously, required an ILP model. Although still optimal in terms of cost, the time taken was significantly worse. In the present work, we describe three significant improvements that are made to the model. These improvements reduced the time taken to optimize the larger networks by orders of magnitude. The time taken to optimize a 150-node network has gone from over $40 \mathrm{~min}$ to $5 \mathrm{~s}$, and a 200-node network, which could not be solved within $24 \mathrm{~h}$ now takes just $70 \mathrm{~s}$.

The improvements consist of tightening the set of constraints used to describe the ILP model. Consider the example shown in figure 2 . The points represent the integer points over which we are trying to optimize. The lines represent the constraints that encompass those integer points. When solving the linear point (LP) relaxation, the entire set $S$ is considered. By introducing the constraint e, we can still capture the same integer points while cutting off a part $\left(S_{2}\right)$ of the linear relaxation. Since a smaller solution space is now considered while solving the LP relaxation, this speeds up the optimization. For each of the three improvements presented, we prove that the newer set of constraints has a linear relaxation that is a strict subset of the linear relaxation of the older set, while maintaining the same set of integer points. In particular, for the tank configuration improvement we show that the newer subset of constraints is as tight as possible, i.e. the linear relaxation has no fractional points. Since the overall model is complex, while discussing each improvement, we consider only a small subset of relevant constraints at a time.

The rest of the paper is structured as follows. In section 2 we describe the optimization problem formulation and the initial model used to solve the problem. In sections 3-5, we describe the three improvements. For each improvement, we first repeat the relevant subset of constraints from the initial model, then provide the new set of constraints of the improved model and then finally prove that the improved model is strictly better than the initial model. In section 6 , we describe an initial attempt at an alternative edge-based

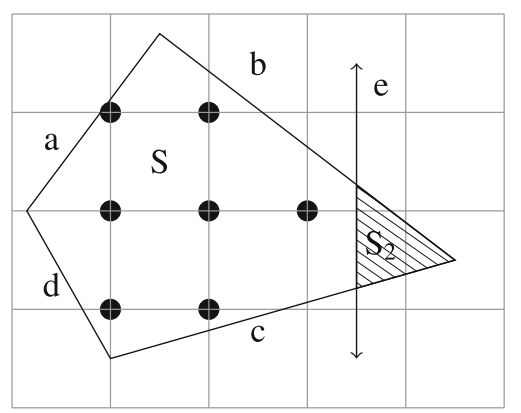

Figure 2. Constraints $a, b, c$ and $d$ describe the area $S$ that represents the linear relaxation of the set of 7 integer points in two dimensions. Introducing the constraint e cuts off the area $S_{2}$ from the linear relaxation, while still maintaining the same set of integer points. approach to modelling the problem. The initial model and the three post-improvement models were tested on eight networks of varying sizes. Section 7 provides the performance details of these tests. Finally, we provide our concluding thoughts in section 8 .

\section{Initial model (model 1)}

As discussed earlier, drinking water distribution networks consist of various components. To optimize the cost of such networks, several inputs must be considered, and for each component, several parameters must be determined. We first explicitly formulate the problem that we are attempting to solve. Then, in section 2.2, we provide details of the initial ILP model used to solve the problem.

\subsection{Problem formulation}

\section{Inputs}

- General: primary/secondary supply hours, minimum/ maximum headloss per $\mathrm{km}$, maximum water speed

- Source node: head

- Node: elevation, water demand, minimum pressure requirement

- Link: start/end node, length

- Existing pipes: start/end node, length, diameter, parallel allowed, roughness

- Commercial pipes: diameter, roughness, cost per unit length

- Tanks: maximum tank heights, tank capacity factor, nodes that must/must not have tanks, capital cost table

- Pumps: minimum pump size, efficiency, design lifetime, capital/energy cost, discount/interest rate, pipes that cannot have pumps

- Valves: location, pressure rating

\section{Outputs}

- Length and diameter of pipe segments for each link

- Partitioning the set of links into primary and secondary network

- Location, height and size of tanks

- Set of nodes being served by each tank

- Location and power of pumps

Objective

- Minimize total capital cost (pipe + tank + pump) and total energy cost (pump)

\section{Constraints}

- Pressure at each node must be at least the minimum pressure specified

- Water demand must be met at each node 


\subsection{Model details}

The pipe diameter selection in the model is represented by the continuous variable $l_{i j}$, which represents the length of the $j^{\text {th }}$ pipe diameter component of the $i^{\text {th }}$ link in the network. This determines the capital cost of the pipes. The tank allocation is represented by the binary variable $s_{n m}$, which is one if the tank at the $n^{\text {th }}$ node in the network provides water to the $m^{\text {th }}$ node in the network. The choice of tank allocation variables fixes the total demand that each tank serves, i.e. the variable $d_{n}$. This in turn determines the capital cost of the tanks. Apart from the cost considerations, each node $n$ must also have its minimum pressure constraint satisfied. The head at each node, $h_{n}$, is dependent on the headloss $h l_{i}$ in the links of the network. This headloss depends on the pipe variables $l_{i j}$ and the tank variables $s_{n m}$ mentioned earlier. In addition, the introduction of pumps/valves increases/decreases the headloss, respectively. The details of the parameters, variables, objective function and constraints of the model are as follows.

\section{Parameters}

- $N L$ : number of links in the network

- NP: number of commercial pipe diameters

- $D_{j}$ : diameter of $j^{\text {th }}$ commercial pipe diameter

- $C_{j}$ : cost per unit length of $j^{\text {th }}$ commercial pipe diameter

- $N N$ : number of nodes in the network

- $N E$ : number of rows in the tank cost table

- $B_{k}$ : base cost of the $k^{\text {th }}$ row of the tank cost table

- $U N_{k}$ : unit cost of the $k^{\text {th }}$ row of the tank cost table

- $U P_{k}$ : upper limit capacity for the $k^{\text {th }}$ row of the tank cost table

- $L O_{k}$ : lower limit capacity for the $k^{\text {th }}$ row of the tank cost table

- $C P$ : capital cost of pumps per unit $\mathrm{kW}$

- EP: energy cost of pumps per unit kWh

- $D F$ : discount factor for the energy cost over the entire scheme lifetime

- $P H$ : number of hours of water supply in the primary network

- $S H$ : number of hours of water supply in the secondary network

- $Y$ : lifetime of scheme in years

- INFR: inflation rate

- INTR: interest rate

- $L_{i}$ : length of the $i^{\text {th }}$ link

- $P R_{n}$ : minimum pressure required at node $n$

- $E_{n}$ : elevation of the $n^{\text {th }}$ node

- $D E_{n}$ : water demand of the $n^{\text {th }}$ node

- $D E$ : total water demand of the network

- $V H_{i}$ : head reduction by valve in $i^{\text {th }}$ link

- $H L_{i j}^{p}$ : headloss for the $j^{\text {th }}$ diameter of the $i^{\text {th }}$ link, if $i$ is part of the primary network

- $H L_{i j}^{s}$ : headloss for the $j^{\text {th }}$ diameter of the $i^{\text {th }}$ link, if $i$ is part of the secondary network
- $F L_{i}^{p}$ : flow in $i^{\text {th }}$ link if $i$ is part of the primary network

- $F L_{i}^{s}$ : flow in $i^{\text {th }}$ link if $i$ is part of the secondary network

- $R_{j}$ : roughness of $j^{\text {th }}$ commercial pipe diameter

- $T_{\text {min }}$ : minimum tank height allowed

- $T_{\max }$ : maximum tank height allowed

- $\rho$ : density of water

- $g$ : acceleration due to gravity

- $\eta$ : efficiency of pump

- $P P_{\min }$ : minimum pump power allowed

- $P P_{\max }$ : maximum pump power allowed

- $A_{n}$ : set of nodes that are ancestors of node $n$ in the network

- $D_{n}$ : set of nodes that are descendants of node $n$ in the network

- $C_{n}$ : set of child nodes of node $n$ in the network

- $P_{n}$ : parent node of node $n$

- $I_{n}$ : incoming link for node $n$

- $O_{n}$ : set of outgoing links from node $n$

\section{Continuous variables}

- $l_{i j}$ : length of the $j^{\text {th }}$ pipe component of the $i^{\text {th }}$ link

- $l_{i j}^{p}$ : length of the $j^{\text {th }}$ pipe component of the $i^{\text {th }}$ link, if link $i$ is part of the primary network

- $h l_{i}$ : total headloss across link $i$

- $d_{n}$ : total demand served by tank at node $n$

- $z_{n k}$ : total demand served by tank at node $n$, if costed by the $k^{\text {th }}$ row of the tank cost table

- $p_{i}$ : power of pump installed at link $i$

- $p_{i}^{p}$ : power of pump installed at link $i$, if link $i$ is part of primary network

- $p_{i}^{s}$ : power of pump installed at link $i$, if link $i$ is part of secondary network

- $p h_{i}$ : head provided by pump at link $i$

- $h_{n}$ : water head at node $n$

- $t_{n}$ : height of tank at node $n$

- $h_{n i}^{\prime}$ : effective head provided to link $i$ by its starting node $n$

\section{Binary variables}

- $e_{n k}: 1$ if tank at $n^{\text {th }}$ node is costed by the $k^{\text {th }}$ row of tank cost table, 0 otherwise

- $f_{i}: 1$ if link $i$ is part of the primary network, 0 if part of the secondary network

- $e s_{n i}: 1$ if source of water for link $i$ is its immediate upstream node $n, 0$ otherwise

- $s_{n m}$ : 1 if tank at node $n$ is source for node $m, 0$ otherwise

- $p e_{i}: 1$ if a pump is installed at link $i, 0$ otherwise.

Objective function: The objective function is simply the sum of capital cost of the pipes, tanks, pumps and valves used in the network. In addition, we also have the operational cost of the pumps. This operational cost is computed as the present value of the total cost over the scheme lifetime: 


$$
\begin{aligned}
O(.)= & \sum_{i=1}^{N L} \sum_{j=1}^{N P} C_{j}\left(D_{j}\right) l_{i j}+\sum_{i=1}^{N L} C P \times p_{i} \\
& +\sum_{n=1}^{N N} \sum_{k=1}^{N E} e_{n k}\left(B_{k}+U N_{k}\left(d_{n}-L O_{k}\right)\right) \\
& +E P \times D F\left(\sum_{i=1}^{N L} P H \times p_{i}^{p}+\sum_{i=1}^{N L} S H \times p_{i}^{S}\right) \\
& \text { where } D F=\sum_{n=1}^{Y}\left(\frac{1+I N F R}{1+I N T R}\right)^{n-1} .
\end{aligned}
$$

\section{Constraints}

- The total length of the pipe diameter segments must equal the total link length:

$$
L_{i}=\sum_{j=1}^{N P} l_{i j}, \quad i=1, \ldots, N L .
$$

- The pressure at each node must exceed the minimum pressure required:

$$
P R_{n} \leq h_{n}-\left(E_{n}+t_{n}\right), \quad n=1, \ldots, N N .
$$

- Across every link $i$ there is headloss $h l_{i}$. This headloss depends on the flow, length and diameter of the pipe chosen. We use the Hazen-Williams equation [19] to calculate the headloss. The headloss across a link also depends on the pump and valve installed across it, if any. The valves are input parameters to the model, since they are manually fixed. The constraints related to the pump head $p h_{i}$ are described further below. The flow through the link depends on whether the link is part of the primary or secondary network:

$$
\begin{gathered}
h l_{i}=\sum_{j=1}^{N P}\left(H L_{i j}^{p} l_{i j}^{p}+H L_{i j}^{s}\left(l_{i j}-l_{i j}^{p}\right)\right)-p h_{i}+V H_{i}, \\
i=1, \ldots, N L . \\
H L_{i j}^{p}=\frac{10.68\left(\frac{F L_{i}^{p}}{R_{j}}\right)^{1.852}}{D_{j}^{4.87}}, \\
i=1, \ldots, N L, \quad j=1, \ldots, N P . \\
H L_{i j}^{s}=\frac{10.68\left(\frac{F L_{i}^{s}}{R_{j}}\right)^{1.852}}{D_{j}^{4.87}}, \\
i=1, \ldots, N L, \quad j=1, \ldots, N P . \\
F L_{i}^{s}=F L_{i}^{p} \frac{P H}{S H}, \quad i=1, \ldots, N L .
\end{gathered}
$$

- The head $h_{n}$ at each node $n$ is calculated by the effective head $h_{m i}^{\prime}$ provided by its parent node $m$ and the headloss $h l_{i}$ across the link connecting two nodes.
The effective head in turn depends on whether the link $i$ has the tank at the starting node $m$ as its source. This is represented by the binary variable $e s_{m i}$ :

$$
\begin{gathered}
h_{n}=h_{m i}^{\prime}-h l_{i}, \\
n=1, \ldots, N N, \quad m=P_{n}, \quad i=I_{n} . \\
h_{m i}^{\prime}=\left(t_{m}+E_{m}\right) e s_{m i}+h_{m}\left(1-e s_{m i}\right), \\
m=1, \ldots, N N, \quad i \in O_{m} . \\
e s_{m i}=s_{m m}\left(1-f_{i}\right), \\
m=1, \ldots, N N, \quad i \in O_{m} .
\end{gathered}
$$

- Next, we look at the constraints related to the tank allocation. The first tank constraint is to ensure that every tank height is between parameters $T_{\min }$ and $T_{\max }$ :

$$
T_{\min } \leq t_{n} \leq T_{\max } .
$$

- We then look at the constraints that model allocation of demand nodes to tanks; $s_{n m}$ is 1 if tank at node $n$ serves the demand of node $m$. If a node $n$ does not serve its own demand, i.e. it is part of a secondary network, then all its downstream nodes will also be part of a secondary network.

$$
s_{m m} \leq s_{n n}, \quad n=1, \ldots, N N, \quad m \in D_{n} .
$$

- If a node $n$ does not serve its own demand, then it cannot serve the demand of its downstream nodes.

$$
s_{n m} \leq s_{n n}, \quad n=1, \ldots, N N, \quad m \in D_{n} .
$$

- For every node $n$, only one upstream node $m$ can serve its demand.

$$
\sum_{m \in A_{n} \cup\{n\}} s_{m n}=1, \quad n=1, \ldots, N N .
$$

- The total demand $d_{n}$ served by node $n$ is the sum of the demands of the downstream nodes that it serves, i.e. all $m$ such that $s_{n m}=1$ :

$$
d_{n}=\sum_{m \in D_{n} \cup\{n\}} s_{n m} \times D E_{m}, \quad n=1, \ldots, N N .
$$

- For a node $n$, its incoming pipe $i$ will have primary flow only if the node serves itself:

$$
f_{i}=s_{n n}, \quad n=1, \ldots, N N, \quad i=I_{n} .
$$

- If a node $n$ serves node $m$, i.e. $s_{n m}=1$, each node $o$ in the path from $n$ to $m$ belongs to a secondary network and therefore cannot serve itself.

$$
\begin{aligned}
& s_{n m} \leq 1-s_{o o}, \quad n=1, \ldots, N N, \quad m \in D_{n}, \\
& \quad o \in D_{n} \cap A_{m} .
\end{aligned}
$$

- Next, we have the constraints that relate the demand that a tank serves to its cost variables $e_{n k}$. Note that we 
require $z_{n k}$ in our objective function to replace the nonlinear term $e_{n k} d_{n}$ :

$$
z_{n k}=e_{n k} d_{n}, \quad n=1, \ldots, N N, \quad k=1, \ldots, N E .
$$

- Since every tank can be costed using exactly one row of the table, the sum of $e_{n k}$ for a given $n$ must be 1 :

$$
\sum_{k=1}^{N E} e_{n k}=1, \quad n=1, \ldots, N N
$$

- Next we have constraints that make sure that the tank capacity $d_{n}$ lies between the minimum and maximum capacity of the selected row of the cost table:

$$
\begin{aligned}
& \text { for } n=1, \ldots, N N, \quad k=1, \ldots, N E \\
& \qquad \begin{array}{c}
L O_{k} e_{n k} \leq d_{n}, \\
D E \times e_{n k}+d_{n} \leq U P_{k}+D E .
\end{array}
\end{aligned}
$$

- Next, we look at constraints related to pumps. The pump power $p_{i}$ relates to the pump head $p h_{i}$ in the following way:

$$
\begin{gathered}
p_{i}=p_{i}^{p}+p_{i}^{s}, \quad i=1, \ldots, N L, \\
p_{i}^{p}=\frac{\left(\rho g \times F L_{i}^{p} \times p h_{i}\right)}{\eta} f_{i}, \quad i=1, \ldots, N L, \\
p_{i}^{s}=\frac{\left(\rho g \times F L_{i}^{s} \times p h_{i}\right)}{\eta}\left(1-f_{i}\right), \quad i=1, \ldots, N L .
\end{gathered}
$$

- Finally, the pump power for each pump must lie between minimum and maximum allowed pump power. This is implemented using the binary variable $p e_{i}$ :

$$
P P_{\text {min }} \times p e_{i} \leq p_{i} \leq P P_{\text {max }} \times p e_{i}, \quad i=1, \ldots, N L .
$$

Note that constraints (18), (23) and (24) contain nonlinear terms. Each of these can be linearized since they are a product of a binary variable and a continuous variable. For the sake of brevity and clarity, the constraints that perform the linearization are not mentioned here. This completes the description of the initial model. Although this model provides optimal results in terms of capital cost, the time taken to solve networks rises rapidly with increased network size. In the next three sections, we go over three improvements made iteratively to this initial model. For each improvement, we first describe the subset of variables and constraints from the initial model that are being considered. We next provide the improved set of constraints. Finally, we prove how the linear relaxation of the improved set is a strict subset of the linear relaxation of the initial set.

\section{Pipe headloss improvement}

\subsection{Initial model}

We focus on a part of the model whose purpose is to determine the pipe diameters chosen for each link in the network. Each link can consist of multiple pipe diameters. Also, each link can be part of the primary network or the secondary network. The headloss across the link depends on these choice of pipe diameters and whether it belongs to the primary or secondary network. The set of variables and parameters used for this purpose are defined as follows. Consider a network of $N L$ links. Let $N P$ be the number of pipe diameters available.

\section{Variables}

$l_{i j}=$ length of the $j^{\text {th }}$ pipe diameter component of link $i$,

$i=1, \ldots, N L, \quad j=1, \ldots, N P$

$l_{i j}^{p}=$ length of the $j^{\text {th }}$ pipe diameter component of link $i$, if link $i$ is part of the primary network, $i=1, \ldots, N L$, $j=1, \ldots, N P$

$h l_{i}=$ headloss across link $i, \quad i=1, \ldots, N L$

$f_{i}=1$ of link $i$ is part of the primary network, 0 if it is part of the secondary network, $\quad i=1, \ldots, N L$.

\section{Parameters}

$L_{i}=$ length of link $i, \quad i=1, \ldots, N L$.

$H L_{i j}^{p}=$ unit headloss for the $j^{\text {th }}$ pipe diameter component of link $i$, if $i$ is part of primary network, $i=1, \ldots, N L, \quad j=1, \ldots, N P$.

$H L_{i j}^{s}=$ unit headloss for the $j^{\text {th }}$ pipe diameter component of link $i$, if $i$ is part of secondary network, $i=1, \ldots, N L, \quad j=1, \ldots, N P$.

\section{Constraints}

The first constraint captures $l_{i j}^{p}$ as a product of $l_{i j}$ and $f_{i}$ :

$$
l_{i j}^{p}=l_{i j} f_{i}, \quad i=1, \ldots, N L, \quad j=1, \ldots, N P .
$$

Equation (26) consists of a product of two variables, and is therefore a nonlinear equation. Fortunately since $f_{i}$ is a binary variable, we can linearize the equation using the following inequalities:

$$
\begin{gathered}
0 \leq l_{i j}^{p}, \quad i=1, \ldots, N L, \quad j=1, \ldots, N P, \\
l_{i j}^{p} \leq L_{i} f_{i}, \quad i=1, \ldots, N L, \quad j=1, \ldots, N P, \\
l_{i j}-L_{i}\left(1-f_{i}\right) \leq l_{i j}^{p}, \quad i=1, \ldots, N L, \quad j=1, \ldots, N P, \\
l_{i j}^{p} \leq l_{i j}, \quad i=1, \ldots, N L, \quad j=1, \ldots, N P .
\end{gathered}
$$

The sum of all pipe diameter components must equal the link length:

$$
\sum_{j=1}^{N P} l_{i j}=L_{i}, \quad i=1, \ldots, N L
$$


Next we have the equation for $h l_{i}$, which is the sum of all headloss components contributed by the different pipe diameter components of link $i$ :

$$
h l_{i}=\sum_{j=1}^{N P} P_{i j} l_{i j}^{p}+\sum_{j=1}^{N P} S_{i j}\left(l_{i j}-l_{i j}^{p}\right), \quad i=1, \ldots, N L .
$$

Finally we have constraints that relate to the bounds for the variables:

$$
\begin{gathered}
l_{i j} \geq 0, \quad i=1, \ldots, N L, \quad j=1, \ldots, N P, \\
f_{i} \in\{0,1\}, \quad i=1, \ldots, N L
\end{gathered}
$$

Since there exists a $l_{i j}^{p}$ for each link and pipe diameter combination in the network, a large number of linear decompositions of equation (26) need to be done. In the next section we show an improved model that has the same feasible $0-1$ set of values but with a tighter LP relaxation, resulting in better performance.

\subsection{Improved model (model 2)}

In order to decompose the product of variables in (26), a large number of constraints need to be added. This is avoided in the new model by not explicitly defining $l_{i j}^{p}$. Instead, its relation to $l_{i j}$ and $f_{i}$ is implicit. In the next section, we show that the new model is better.

\section{Variables}

We introduce one new variable, which is similar to $l_{i j}^{p}$, but for the secondary network:

$l_{i j}^{s}=$ length of the $j^{\text {th }}$ pipe diameter component of link $i$, if link $i$ is part of the secondary network, and 0 if link $i$ is part of the primary network, $\quad i=1, \ldots, N L, \quad j=1, \ldots, N P$. Constraints

The first constraint simply states that $l_{i j}$ is the sum of the primary and secondary components, i.e. $l_{i j}^{p}$ and $l_{i j}^{s}$, respectively:

$$
l_{i j}=l_{i j}^{p}+l_{i j}^{s}, \quad i=1, \ldots, N L, \quad j=1, \ldots, N P .
$$

For a given link $i$, either all $l_{i j}^{p}$ are 0 or all $l_{i j}^{s}$ are 0 , depending on the value of $f_{i}$. And the sum of the non-zero components must equal the length of the link $L_{i}$. The first two constraints of the new model capture this:

$$
\begin{gathered}
\sum_{j=1}^{N P} l_{i j}^{p}=L_{i} f_{i}, \quad i=1, \ldots, N L, \\
\sum_{j=1}^{N P} l_{i j}^{s}=L_{i}\left(1-f_{i}\right), \quad i=1, \ldots, N L .
\end{gathered}
$$

Next we have the equation for $h l_{i}$, which is the sum of all headloss components contributed by the different pipe diameter components of link $i$. For the new model, we equivalently use $l_{i j}^{s}$ instead of $l_{i j}-l_{i j}^{p}$ due to equation (35):

$$
h l_{i}=\sum_{j=1}^{N P} P_{i j} l_{i j}^{p}+\sum_{j=1}^{N P} S_{i j} l_{i j}^{s}, \quad i=1, \ldots, N L .
$$

Finally, as before we have the bounds for the variables:

$$
\begin{gathered}
l_{i j} \geq 0, \quad i=1, \ldots, N L, \quad j=1, \ldots, N P, \\
l_{i j}^{p} \geq 0, \quad i=1, \ldots, N L, \quad j=1, \ldots, N P, \\
l_{i j}^{s} \geq 0, \quad i=1, \ldots, N L, \quad j=1, \ldots, N P, \\
f_{i} \in\{0,1\}, \quad i=1, \ldots, N L .
\end{gathered}
$$

We now prove that the improved model is tighter than the initial model, that is the linear relaxation of the improved model is a strict subset of the linear relaxation of the initial model. Let $S_{1}$ be the set of points belonging to the initial model and $S_{2}$ be the set of points belonging to the improved model. Let $R_{1}$ and $R_{2}$ be the set of feasible points of the LP relaxations of $S_{1}$ and $S_{2}$, respectively. Both $R_{1}$ and $R_{2}$ are defined by the same set of constraints that describe the initial sets $S_{1}$ and $S_{2}$, except for the constraint (34), which refers to the binary nature of $f_{i}$. Instead, the continuous bounds for $f_{i}$ is defined as follows:

$$
0 \leq f_{i} \leq 1, \quad i=1, \ldots, N L .
$$

Proposition $1 R_{2}$ is a strict subset of $R_{1}$, i.e. $R_{2} \subset R_{1}$.

We prove that $R_{2}$ is a strict subset of $R_{1}$ in two steps. First, we show that $R_{2}$ is a subset of $R_{1}$ and then we find a point in $R_{1}$ that is not in $R_{2}$.

Consider a point $P \in R_{2}$. It satisfies the constraints (27), (33) and (35)-(40). We prove that it also lies in $R_{1}$ by showing that it satisfies the constraints (27)-(33) and (40). Constraints (27), (33) and (40) are trivially satisfied since they are common for both sets.

$$
\begin{aligned}
& \text { For } i=1, \ldots, N L, \quad j=1, \ldots, N P \\
& \operatorname{proving}(28): \quad l_{i j}^{p} \leq L_{i} f_{i} \\
& \sum_{j=1}^{N P} l_{i j}^{p}=L_{i} f_{i} \\
& \equiv \quad\left\{\operatorname{using} l_{i j}^{p} \geq 0(27)\right\} \\
& \quad l_{i j}^{p} \leq L_{i} f_{i} .
\end{aligned}
$$

Hence satisfied. 


$$
\begin{aligned}
& \operatorname{proving}(29): \quad l_{i j}-L_{i}\left(1-f_{i}\right) \leq l_{i j}^{p} \\
& \sum_{j=1}^{N P} l_{i j}^{s}=L_{i}\left(1-f_{i}\right) \\
\Rightarrow & \left\{\text { using } l_{i j}^{s} \geq 0(39)\right\} \\
& l_{i j}^{s} \leq L_{i}\left(1-f_{i}\right) \\
\equiv & \left\{\text { using } l_{i j}=l_{i j}^{p}+l_{i j}^{s}(35)\right\} \\
& l_{i j}-l_{i j}^{p} \leq L_{i}\left(1-f_{i}\right) \\
\equiv & \{\text { rearranging }\} \\
& l_{i j}-L_{i}\left(1-f_{i}\right) \leq l_{i j}^{p} .
\end{aligned}
$$

Hence satisfied.

$$
\begin{aligned}
& \text { Proving (30): } l_{i j}^{p} \leq l_{i j} \\
& 0 \leq l_{i j}^{s} \\
\equiv & \left\{\text { using } l_{i j}=l_{i j}^{p}+l_{i j}^{s}(35)\right\} \\
& 0 \leq l_{i j}-l_{i j}^{p} \\
\equiv & \{\text { rearranging }\} \\
& l_{i j}^{p} \leq l_{i j} .
\end{aligned}
$$

Hence satisfied.

$$
\begin{gathered}
\text { Proving }(31): \sum_{j=1}^{N P} l_{i j}=L_{i} \\
\sum_{j=1}^{N P} l_{i j}^{p}=L_{i} f_{i} \\
\sum_{j=1}^{N P} l_{i j}^{s}=L_{i}\left(1-f_{i}\right) \\
\left.\equiv \sum_{j=1} \text { adding equations }\right\} \\
\left.\equiv l_{i j}^{N P}+l_{i j}^{s}\right)=L_{i} f_{i}+L_{i}\left(1-f_{i}\right) \\
\sum_{j=1}^{N P} l_{i j}=L_{i} .
\end{gathered}
$$

Hence satisfied.

$$
\begin{aligned}
& \operatorname{Proving}(32): h l_{i}=\sum_{j=1}^{N P} P_{i j} l_{i j}^{p}+\sum_{j=1}^{N E} S_{i j}\left(l_{i j}-l_{i j}^{s}\right) \\
& h l_{i}=\sum_{j=1}^{N P} P_{i j} l_{i j}^{p}+\sum_{j=1}^{N P} S_{i j} l_{i j}^{s} \\
& \equiv\left\{\operatorname{using} l_{i j}=l_{i j}^{p}+l_{i j}^{s}(35)\right\} \\
& h l_{i}=\sum_{j=1}^{N P} P_{i j} l_{i j}^{p}+\sum_{j=1}^{N E} S_{i j}\left(l_{i j}-l_{i j}^{s}\right) .
\end{aligned}
$$

$$
\text { (27)-(33) and (40). Therefore } R_{2} \subseteq R_{1} \text {. }
$$

Therefore point $P \in R_{1}$, since it satisfies the constraints

Next we find a point $Q$ such that $Q \in R_{1}$ and $Q \notin R_{2}$. Take point $Q\left(l, l^{p}, l^{s}, h l, f\right)=([L / 2, L / 2], \quad[L / 2, L / 2]$, $[0,0], \quad L, \quad 1 / 2)$. Here $(n, m)=(1,2)$ and $(L, P, S)=$ $(L,[1,1],[1,1])$ where $L \geq 0 . Q \in R_{1}$ since it satisfies the constraints (27)-(33) and (40). Also, point $Q \notin R_{2}$ since it does not satisfy equation (37).

\section{Tank cost improvement}

\subsection{Initial model}

We next focus on a part of the model whose purpose is to determine the capital cost of each tank in the network. The tank cost is a piecewise-linear function. An example is shown in figure 3 . We need to determine which row in the tank cost table the tank capacity falls in. Each row in the tank cost table has minimum and maximum capacity values. If the tank capacity is within these values, then that row is used to compute the tank's cost. In the example considered, if a tank capacity of 51 is to be built, then the first row of the cost table will be used, since its capacity range is $0-10$. Binary variables are used to capture, for each tank, the row in the cost table that is chosen to compute the cost. The set of variables and parameters used for this purpose are defined as follows. Consider a network of $n$ locations. Let $m$ be the number of linear components of the piecewise-linear cost of construction of a tank.

\section{Variables}

$e_{n k}=1$ if the tank at location $n$ is costed using the $k^{\text {th }}$ row of the tank cost table, $\quad n=1, \ldots, N N, \quad k=1, \ldots, N E$.

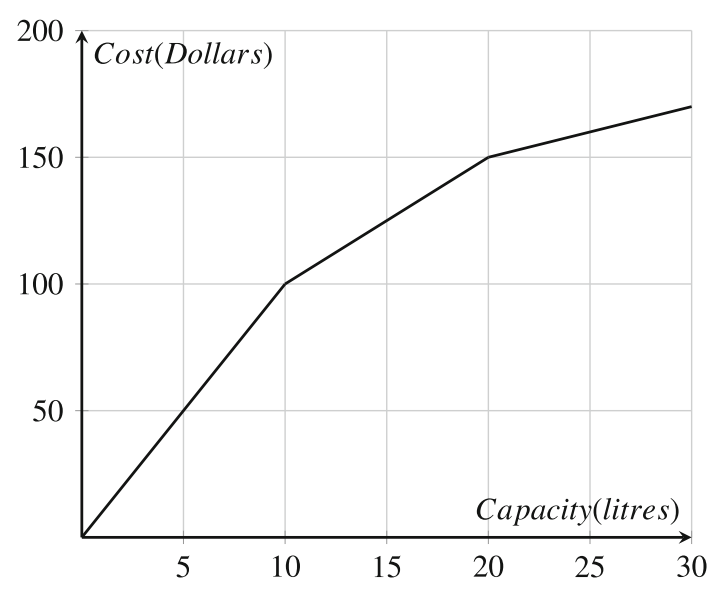

Figure 3. Graph of the cost of a tank vs its capacity. It is a concave piecewise-linear function.

Hence satisfied. 
$z_{n k}=$ capacity of the tank at location $n$ if it is costed using the $k^{\text {th }}$ row of the tank cost table, 0 otherwise, $n=1, \ldots, N N, \quad k=1, \ldots, N E$.

$d_{n}=$ capacity of tank at location $n, \quad n=1, \ldots, N N$.

\section{Parameters}

$L O_{k}=$ minimum capacity that the $k^{\text {th }}$ row of the tank cost table can satisfy, $k=1, \ldots, N E$.

$U P_{k}=$ maximum capacity that the $k^{\text {th }}$ row of the tank cost table can satisfy, $k=1, \ldots, N E$.

$D E$ = value of the total water demand in the network, where $D E \geq U P_{k}, \quad k=1, \ldots, N E$.

\section{Constraints}

The first constraint relates the tank capacity corresponding to the $k^{\text {th }}$ row as a product of the tank capacity and the binary choice variable $e_{n k}$ :

$$
z_{n k}=e_{n k} d_{n}, \quad n=1, \ldots, N N, \quad k=1, \ldots, N E .
$$

Since equation (41) consists of a product of two variables, it is a nonlinear equation. We linearize the equation using the following inequalities:

$$
\begin{gathered}
0 \leq z_{n k}, n=1, \ldots, N N, \quad k=1, \ldots, N E, \quad \\
z_{n k} \leq D E e_{n k}, n=1, \ldots, N N, \quad k=1, \ldots, N E, \quad(41 . \mathrm{b}) \\
d_{n}-D E\left(1-e_{n k}\right) \leq z_{n k}, n=1, \ldots, N N, \quad k=1, \ldots, N E,
\end{gathered}
$$

$$
z_{n k} \leq d_{n}, n=1, \ldots, N N, \quad k=1, \ldots, N E .
$$

Since every tank can be costed using exactly one row, the sum of $e_{n k}$ for a given $n$ must be 1 :

$$
\sum_{k=1}^{N E} e_{n k}=1, \quad n=1, \ldots, N N
$$

Next, we have constraints that make sure that the tank capacity $d_{n}$ lies between the minimum and maximum capacity of the selected row of the cost table:

$$
L O_{k} \times e_{n k} \leq d_{n}, n=1, \ldots, N N, k=1, \ldots, N E,
$$

$D E \times e_{n k}+d_{n} \leq U P_{n}+D E, n=1, \ldots, N N, k=1, \ldots, N E$.

Finally, we have constraints that relate to the bounds for the variables:

$$
\begin{gathered}
D E \geq d_{n}, \quad n=1, \ldots, N N, \\
d_{n} \geq 0, \quad n=1, \ldots, N N,
\end{gathered}
$$

$$
e_{n k} \in\{0,1\}, \quad n=1, \ldots, N N, \quad k=1, \ldots, N E .
$$

Since there exists a $z_{n k}$ for each tank and row of cost table combination, a large number of linear decompositions of equation (41) need to be done. This results in poor performance of the model. In the next section we show an improved model that has the same feasible $0-1$ set of values but with a tighter LP bound, resulting in better performance.

\subsection{Improved model (model 3)}

As discussed in the previous section, the main issue with the old model is equation (41), where $z_{n k}$ is expressed as a product of two variables. In order to decompose the variables, a large number of constraints need to be added. This is avoided in the new model by not explicitly defining $z_{n k}$. Instead, its relation to $e_{n k}$ and $d_{n}$ is implicit. In the next section we first show that the new model is better in that it has a tighter LP bound than the old model, and then we go on to show that the LP for the new model has tight solutions.

The variables remain the same for the new model. The first two inequalities of the model provide the bounds for $z_{n k}$ in terms of $e_{n k}$ and the minimum $\left(L O_{k}\right)$ and maximum $\left(U P_{k}\right)$ capacities for each row of the cost table:

$$
\begin{aligned}
& L O_{k} e_{n k} \leq z_{n k}, \quad n=1, \ldots, N N, \quad k=1, \ldots, N E, \\
& z_{n k} \leq U P_{k} e_{n k}, \quad n=1, \ldots, N N, \quad k=1, \ldots, N E .
\end{aligned}
$$

The next equation for the model remains unchanged; it represents the fact that each row of the cost table is chosen exactly once for each tank:

$$
\sum_{k=1}^{N E} e_{n k}=1, \quad n=1, \ldots, N N
$$

Next, we have a similar equation but this time related to the variable $z_{n k}$. The sum of all $z_{n k}$ values for a given tank must equal $d_{n}$ :

$$
\sum_{k=1}^{N E} z_{n k}=d_{n}, \quad n=1, \ldots, N N
$$

In fact, along with the previous three equations of the model, one can infer that exactly one of the $z_{n k}$ values will be nonzero for a specific tank and therefore will be equal to $d_{n}$. This therefore captures the nonlinear constraint that equation (41) of the old model captured. The remaining constraints relate to the bounds for the variables:

$$
\begin{gathered}
D E \geq d_{n}, \quad n=1, \ldots, N N, \\
e_{n k} \in\{0,1\}, \quad n=1, \ldots, N N, \quad k=1, \ldots, N E,
\end{gathered}
$$




$$
\begin{gathered}
d_{n} \geq 0, \quad n=1, \ldots, N N, \\
z_{n k} \geq 0, \quad n=1, \ldots, N N, \quad k=1, \ldots, N E .
\end{gathered}
$$

Let $S_{1}$ be the set of points belonging to the old model and $S_{2}$ be the set of points belonging to the new model. Let $R_{1}$ and $R_{2}$ be the set of feasible points of the LP relaxations of $S_{1}$ and $S_{2}$, respectively. The continuous bounds for $e_{n k}$ are defined as follows:

$$
0 \leq e_{n k} \leq 1, \quad n=1, \ldots, N N, \quad k=1, \ldots, N E
$$

As in section 3, we prove that the LP relaxation of the new model is tighter than the LP relaxation of the old model. We then go on to show that $R_{2}$ has no fractional corner points, and thus cannot be tightened further.

Proposition $2 R_{2}$ is a strict subset of $R_{1}$, i.e. $R_{2} \subset R_{1}$.

As in Proposition 1, we prove that $R_{2}$ is a strict subset of $R_{1}$ in two steps. First we show that $R_{2}$ is a subset of $R_{1}$, and then we show that $R_{2}$ is not equal to $R_{1}$.

Consider a point $P \in R_{2}$. It satisfies the constraints (41.a), (42) and (45)-(51). We prove that it also lies in $R_{1}$ by showing that it satisfies the constraints (41.a)-(46) and (51). Constraints (41.a), (42), (45), (46) and (51) are trivially satisfied since they are common for both sets.

$$
\begin{aligned}
& \text { For } \quad n=1, \ldots, N N, \quad k=1, \ldots, N E \\
& \text { proving }(41 . b): \quad z_{n k} \leq D E e_{n k} \\
& z_{n k} \leq U P_{k} e_{n k} \\
& \Rightarrow \quad\left\{\text { using } D E \geq U P_{k} \text { (definition) }\right\} \\
& z_{n k} \leq D E e_{n k} \text {. } \\
& \text { Hence satisfied. } \\
& \text { Proving (41.c) : } \quad d_{n}-D E\left(1-e_{n k}\right) \leq z_{n k} \\
& \sum_{k^{\prime}=1}^{N E} z_{n k^{\prime}}=d_{n} \\
& \equiv\{\text { splitting sum }\} \\
& z_{n k}+\sum_{k^{\prime}=1, k^{\prime} \neq k}^{N E} z_{n k^{\prime}}=d_{n} \\
& \equiv\{\text { rearranging }\} \\
& \sum_{k^{\prime}=1, k^{\prime} \neq k}^{N E} z_{n k^{\prime}}=d_{n}-z_{n k} \\
& e_{n k}+\sum_{k^{\prime}=1, k^{\prime} \neq k}^{N E} e_{n k^{\prime}}=1 \\
& \equiv \quad\{\text { rearranging }\}
\end{aligned}
$$

$$
\begin{aligned}
\Rightarrow \quad\left\{\text { sum over } k^{\prime}\right\} & \\
& \sum_{k^{\prime}=1, k^{\prime} \neq k}^{N E} z_{n k^{\prime}} \leq D E \sum_{k^{\prime}=1, k^{\prime} \neq k}^{N E} e_{n k^{\prime}}
\end{aligned}
$$$$
\equiv\{\operatorname{using}(52),(53)\}
$$$$
d_{n}-z_{n k} \leq D E\left(1-e_{n k}\right)
$$$$
\equiv\{\text { rearranging }\}
$$$$
d_{n}-D E\left(1-e_{n k}\right) \leq z_{n k} \text {. }
$$

Hence satisfied.

$$
\begin{gathered}
\text { Proving (41.d) : } \quad z_{n k} \leq d_{n} \\
\sum_{k=1}^{N E} z_{n k}=d_{n}, \\
\Rightarrow \quad\left\{\text { using } 0 \leq z_{n k}(41 . a)\right\} \\
z_{n k} \leq d_{n} .
\end{gathered}
$$

Hence satisfied.

$$
\begin{aligned}
& \text { Proving }(43): \quad L O_{k} \times e_{n k} \leq d_{n} \\
& L O_{k} \times e_{n k} \leq z_{n k} \\
\Rightarrow \quad & \left\{\operatorname{using} z_{n k} \leq d_{n}(41 . d)\right\} \\
& L O_{k} \times e_{n k} \leq d_{n} .
\end{aligned}
$$$$
\text { Hence satisfied. }
$$

$$
\begin{aligned}
& \text { Proving }(44): \quad d_{n}+D E \times e_{n k} \leq U P_{k}+D E \\
& d_{n}-D E\left(1-e_{n k}\right) \leq z_{n k} \\
\Rightarrow \quad & \left\{\text { using } z_{n k} \leq U P_{k} \times e_{n k}(49)\right\} \\
& d_{n}-D E+D E \times e_{n k} \leq U P_{k} \times e_{n k} \\
\Rightarrow \quad & \left\{\text { using } 0 \leq e_{n k} \leq 1(51)\right\} \\
& d_{n}-D E+D E \times e_{n k} \leq U P_{k} \\
\equiv \quad & \{\text { rearranging }\} \\
& d_{n}+D E \times e_{n k} \leq U P_{k}+D E .
\end{aligned}
$$

Hence satisfied.

Therefore point $P \in R_{1}$ and $R_{2} \subseteq R_{1}$.

Next we find a point $Q$ such that $Q \in R_{1}$ and $Q \notin R_{2}$. Take a point $Q(z, e, d)=([d, d],[1 / 2,1 / 2], d)$. Here $(n, m)=(1,2),\left(L O_{k}, U P_{k}, D E\right)=([0, d],[d, 2 d], 2 d)$ where $d \geq 0$. $Q \in R_{1}$ since it satisfies the constraints (41.a)-(46) and (51). Also point $Q \notin R_{2}$ since it violates equation (50).

We next show that in fact $R_{2}$ has the tightest relaxation possible by showing that a point with fraction value for $e_{n k}$ will never be a corner point.

Proposition 3 If point $P \in R_{2}$ has a fractional value for $e_{n k}, P$ cannot be a corner point of $R_{2}$. 
Proof Consider a point $P(z, e, d) \in R_{2}$ with at least one fractional value for $e_{n k}$, i.e. $0<e_{n^{\prime} k^{\prime}}<1$ for some $n^{\prime}, k^{\prime}$. Let $e_{n^{\prime} k^{\prime}}=t$. Construct another point $P_{1}$ that has the same components of $P$ for $n \neq n^{\prime}$. For $n=n^{\prime}$, take $(z, e, d)$ as follows:

$$
\text { For } \begin{aligned}
k & =1, \ldots, N E \\
z_{n^{\prime} k^{\prime}} & =0 \\
z_{n^{\prime} k} & =\frac{z_{n^{\prime} k}^{P}}{1-t}, \quad \text { for } k \neq k^{\prime} \\
e_{n^{\prime} k^{\prime}} & =0 \\
e_{n^{\prime} k} & =\frac{e_{n^{\prime} k}^{P}}{1-t}, \quad \text { for } k \neq k^{\prime} \\
d_{k^{\prime}} & =\frac{d_{n^{\prime}}^{P}-z_{n^{\prime} k^{\prime}}^{P}}{1-t} .
\end{aligned}
$$

Here, $z_{n^{\prime} k^{\prime}}^{P}, e_{n^{\prime} k^{\prime}}^{P}, d_{n^{\prime}}^{P}$ are the corresponding values of point $P$. We show that $P_{1} \in R_{2}$ since it satisfies all the constraints:

48:

$$
\begin{aligned}
& L O_{k^{\prime}} \times e_{n^{\prime} k^{\prime}} \leq z_{n^{\prime} k^{\prime}} \\
& \equiv L O_{k^{\prime}} \times 0 \leq 0 \\
& L O_{k} \times e_{n^{\prime} k} \leq z_{n^{\prime} k}, \quad k \neq k^{\prime} \\
& \equiv L O_{k} \frac{e_{n^{\prime} k}^{P}}{1-t} \leq \frac{z_{n^{\prime} k}^{P}}{1-t}, \quad k \neq k^{\prime} \\
& \equiv L O_{k} \times e_{n^{\prime} k}^{P} \leq z_{n^{\prime} k}^{P}, \quad k \neq k^{\prime} \\
& \text { Satisfied since } P \in R_{2} \text {. } \\
& \text { 49: } \\
& z_{n^{\prime} k^{\prime}} \leq U P_{k^{\prime}} \times e_{n^{\prime} k^{\prime}} \\
& \equiv 0 \leq U P_{k^{\prime}} \times 0 \\
& z_{n^{\prime} k} \leq U P_{k} \times e_{n^{\prime} k}, \quad k \neq k^{\prime} \\
& \equiv \frac{z_{n^{\prime} k}^{P}}{1-t} \leq U P_{k} \frac{e_{n^{\prime} k}^{P}}{1-t}, \quad k \neq k^{\prime} \\
& \equiv z_{n^{\prime} k}^{P} \leq U P_{k} \times e_{n^{\prime} k}^{P}, \quad k \neq k^{\prime} \quad(0<t<1) \text {. } \\
& \text { Satisfied since } P \in R_{2} \text {. } \\
& \text { 42: } \\
& \sum_{k=1}^{N E} e_{n^{\prime} k} \\
& =0+\sum_{k=1, k \neq k^{\prime}}^{N E} e_{n^{\prime} k} \\
& =\sum_{k=1, k \neq k^{\prime}}^{N E} \frac{e_{n^{\prime} k}^{P}}{1-t} \\
& =\frac{1-e_{n^{\prime} k^{\prime}}^{P}}{1-t} \\
& =\frac{1-t}{1-t} \\
& =1 \\
& \text { 50: } \\
& \sum_{k=1}^{N E} z_{n^{\prime} k} \\
& =0+\sum_{k=1, k \neq k^{\prime}}^{N E} z_{n^{\prime} k} \\
& =\sum_{k=1, k \neq k^{\prime}}^{N E} \frac{z_{n^{\prime} k}^{P}}{1-t} \\
& (0<t<1) \text {. }
\end{aligned}
$$

$$
\begin{gathered}
=\frac{d_{n^{\prime}}^{P}-e_{n^{\prime} k^{\prime}}^{P}}{1-t} \\
=d_{n^{\prime}}
\end{gathered}
$$

51:

$$
\begin{aligned}
& \sum_{k=1}^{N E} e_{n^{\prime} k}^{P}=1 \\
\equiv & e_{n^{\prime} k^{\prime}}^{P}+\sum_{k=1, k \neq k^{\prime}}^{N E} e_{n^{\prime} k}^{P}=1 \\
\equiv & t+\sum_{k=1, k \neq k^{\prime}}^{N E} e_{n^{\prime} k}^{P}=1 \\
\equiv & \sum_{k=1, k \neq k^{\prime}}^{N E} e_{n^{\prime} k}^{P}=1-t \\
\equiv & 0 \leq e_{n^{\prime} k}^{P} \leq 1-t, \quad k \neq k^{\prime}
\end{aligned}
$$

$$
\begin{aligned}
& \equiv 0 \leq \frac{e_{n^{\prime} k}^{P}}{1-t} \leq 1, \quad k \neq k^{\prime} \quad(1-t>0) \\
& \equiv 0 \leq e_{n^{\prime} k} \leq 1, \quad k \neq k^{\prime} \quad \text { (definition). }
\end{aligned}
$$

46:

$$
\begin{array}{cc}
\sum_{k=1}^{N E} z_{n^{\prime} k}^{P}=d_{n^{\prime}}^{P} & \left(P \in R_{2}\right) \\
\equiv z_{n^{\prime} k^{\prime}}^{P}+\sum_{k=1, k \neq k^{\prime}}^{N E} z_{n^{\prime} k}^{P}=d_{n^{\prime}}^{P} & (\text { splitting sum) } \\
\equiv \sum_{k=1, k \neq k^{\prime}}^{N E} z_{n^{\prime} k}^{P}=d_{n^{\prime}}^{P}-z_{n^{\prime} k^{\prime}}^{P} & (\text { rearranging) } \\
\equiv d_{n^{\prime}}^{P}-z_{n^{\prime} k^{\prime}}^{P} \geq 0 & \left(z_{n^{\prime} k}^{P} \geq 0\right) \\
\equiv \frac{d_{n^{\prime}}^{P}-z_{n^{\prime} k^{\prime}}^{P} \geq 0}{1-t} \geq 0 & (1-t>0) \\
\equiv d_{n^{\prime}} \geq 0 & \text { (definition). }
\end{array}
$$

41.a:

$$
\begin{array}{rlrl} 
& z_{n^{\prime} k}^{P} \geq 0, \quad k \neq k^{\prime} & \left(P \in R_{2}\right) \\
\equiv \frac{z_{n^{\prime} k}^{P} \geq 0, \quad k \neq k^{\prime}}{1-t} & (1-t>0) \\
\equiv z_{n^{\prime} k} \geq 0, \quad k \neq k^{\prime} & \text { (definition). }
\end{array}
$$

Therefore $P_{1} \in R_{2}$.

Similar to $P_{1}$, construct point $P_{2}$ having the same components as $P$ for $n \neq n^{\prime}$. For $n=n^{\prime}$ take $(z, e, d)$ as follows:

$$
\text { for } \begin{aligned}
k & =1, \ldots, N E \\
z_{n^{\prime} k^{\prime}} & =\frac{z_{n^{\prime} k^{\prime}}^{P}}{t} \\
z_{n^{\prime} k} & =0 \quad \text { for } k \neq k^{\prime} \\
e_{n^{\prime} k^{\prime}} & =1 \\
e_{n^{\prime} k} & =0 \quad \text { for } k \neq k^{\prime} \\
d_{n^{\prime}} & =\frac{z_{n^{\prime} k^{\prime}}^{P}}{t} .
\end{aligned}
$$


As before, $z_{n^{\prime} k^{\prime}}^{P}, e_{n^{\prime} k^{\prime}}^{P}, d_{n^{\prime}}^{P}$ are the corresponding values of point $P$. With similar arguments as before, we see that $P_{2} \in R_{2}$. Finally

$$
\begin{aligned}
P_{1} & =\left(\left(\frac{z_{n^{\prime} k}^{P}}{1-t}, 0\right),\left(\frac{e_{n^{\prime} k}^{P}}{1-t}, 0\right), \frac{d_{i}^{\prime P}-z_{n^{\prime} k^{\prime}}^{P}}{1-t}\right) \\
P_{2} & =\left(\left(0, \frac{z_{n^{\prime} k^{\prime}}^{P}}{t}\right),(0,1), \frac{z_{n^{\prime} k^{\prime}}^{P}}{t}\right) \\
& \Rightarrow P_{1}(1-t)+P_{2} t=\left(\left(z_{n^{\prime} k}^{P}, z_{n^{\prime} k^{\prime}}^{P}\right),\left(e_{n^{\prime} k}^{P}, t\right), d_{i}^{\prime P}\right) \\
& =P .
\end{aligned}
$$

Since $P$ can be represented as a linear combination of two other points belonging to $R_{2}, P$ cannot be a corner point of $R_{2}$. This implies that LP relaxation $\left(R_{2}\right)$ of the new model will provide only integer solutions. Therefore, the new model has a tight relaxation.

\section{Tank configuration improvement}

For a given network of nodes and links, one aspect of the problem is to determine the location of tanks and the set of downstream nodes that are to be served by each tank. We need a set of constraints to model a valid network configuration. For a given branched network layout with a single source, a valid network configuration is one in which

1. Each node needs to be provided water, by exactly one of its ancestors (including itself).

2. If a node $n$ provides water to itself, i.e. it has a tank, only then can it provide water to its descendants.

3 . If a node $n$ gets water from another tank, then all its descendants cannot get water from themselves.

4. If node $n$ provides water to one of its descendants $k$, then the nodes along the path connecting them cannot serve themselves.

In the following section we repeat the set of constraints that model such a network as laid out in section 2 . We then show that the model is not tight, i.e. its linear relaxation is not guaranteed to have integral corner points. In section 5.2, we then describe an improved model and prove its tightness. In section 6 , we describe an alternate edge-based approach to model the network.

\subsection{Initial model}

Consider a tree network of $n$ nodes.

Parameters

$A_{n}=$ set of ancestor nodes of node $n, n=1, \ldots, N N$.

$D_{n}=$ set of descendant nodes of node $n, n=1, \ldots, N N$.

$C_{n}=$ set of child nodes of node $n, n=1, \ldots, N N$.

$P_{n}=$ parent node of node $n, n=1, \ldots, N N$.

Variables $s_{n m}=1$ if tank at $n^{\text {th }}$ node serves the demand of $m^{\text {th }}$ node, $\quad n=1, \ldots, N N, \quad m \in D_{n} \cup\{n\}$.

Constraints

We can use the following set of constraints to describe the set of valid network configurations as described earlier:

$$
\begin{aligned}
s_{m m} \leq s_{n n}, \quad n=1, \ldots, N N, \quad m \in D_{n}, \\
s_{n m} \leq s_{n n}, \quad n=1, \ldots, N N, \quad m \in D_{n}, \\
\sum_{m} s_{m n}=1, \quad n=1, \ldots, N N, \quad m \in A_{n} \cup\{n\}, \\
s_{n m} \leq 1-s_{o o}, \quad n=1, \ldots, N N, \quad m \in D_{n}, \quad o \in D_{n} \cup A_{m}, \\
s_{n m} \in\{0,1\}, \quad n=1, \ldots, N N, \quad m \in D_{n} \cup\{n\} .
\end{aligned}
$$

Proposition 4 The linear relaxation of $S$ is not tight.

Proof Let the linear relaxation of set $S$ be $R$. Instead of constraint (58), we will have the following constraint:

$$
0 \leq s_{n m} \leq 1, \quad n=1, \ldots, N N, \quad m \in D_{n} \cup\{n\} .
$$

Consider a small network of 3 nodes with node 1 as root, node 2 as child of node 1 and node 3 as child of node 2 . For a point to belong to $R$, the following constraints must be met:

$$
\begin{gathered}
s_{22} \leq s_{11}, \\
s_{33} \leq s_{11}, \\
s_{33} \leq s_{22}, \\
s_{12} \leq s_{11}, \\
s_{13} \leq s_{11}, \\
s_{23} \leq s_{22}, \\
s_{11}=1, \\
s_{12}+s_{22}=1, \\
s_{13}+s_{23}+s_{33}=1, \\
s_{13} \leq 1-s_{22}, \\
0 \leq s_{11}, s_{12}, s_{13}, s_{22}, s_{23}, s_{33} \leq 1 .
\end{gathered}
$$

Since $s_{11}=1$, we replace its value in the constraints and replace repeating constraints to get the following set:

$$
s_{33} \leq s_{22},
$$




$$
\begin{gathered}
s_{23} \leq s_{22}, \\
s_{11}=1, \\
s_{12}+s_{22}=1, \\
s_{13}+s_{23}+s_{33}=1, \\
s_{13} \leq 1-s_{22}, \\
0 \leq s_{12}, s_{13}, s_{22}, s_{23}, s_{33} \leq 1 .
\end{gathered}
$$

\section{Consider a point $P$ defined as}

$P\left\{s_{11}, s_{12}, s_{13}, s_{22}, s_{23}, s_{33}\right\}=\left\{1, \frac{1}{2}, 0, \frac{1}{2}, \frac{1}{2}, \frac{1}{2}\right\}$. Since it satisfies all the constraints, $P \in R$. We now show that $\mathrm{P}$ cannot be described as a linear combination of two distinct points that belong to $R$.

Consider two points $Q_{1}, Q_{2} \in R$ such that

$$
\begin{array}{ccc} 
& P=t Q_{1}+(1-t) Q_{2}, & 0<t<1 \\
& s_{11}^{Q_{1}}=s_{11}^{Q_{2}}=1 & \{56 . a\} \\
& s_{13}^{P}=0 & \{\text { definition }\} \\
& s_{13}^{Q_{1}}=s_{13}^{Q_{2}}=0 & \{59 . c\} \\
\Rightarrow & s_{33}+s_{23} \leq 2 s_{22} & \text { adding } 54 . c \text { cand } 55 . c\} \\
\Rightarrow & 1-s_{13}^{Q_{1}} \leq 2 s_{22}^{Q_{1}} & \{56 . c\} \\
\Rightarrow & \frac{1}{2} \leq s_{22}^{Q_{1}} & \{60\} \\
\Rightarrow & \frac{1}{2} \leq s_{22}^{Q_{2}} & \{\text { Similarly }\} \\
\Rightarrow & s_{22}^{Q_{1}}=s_{22}^{Q_{2}}=\frac{1}{2} & \left\{s_{22}^{P}=\frac{1}{2}\right\} \\
& s_{12}+s_{22}=1 & \{56 . b\} \\
& s_{12}^{Q_{1}}=s_{12}^{Q_{2}}=\frac{1}{2} & \{61\} \\
\Rightarrow & s_{33} \leq s_{22} & \{54 . c\} \\
& s_{33}^{Q_{1}} \leq \frac{1}{2} & \{61\} \\
\Rightarrow & s_{23} \leq s_{22} & \{55 . c\} \\
\Rightarrow & s_{23}^{Q_{1}} \leq \frac{1}{2} & \{61\} \\
& s_{23}^{Q_{1}}=s_{33}^{Q_{1}}=\frac{1}{2} & \{56 . c\} \\
& s_{23}^{Q_{2}}=s_{33}^{Q_{2}}=\frac{1}{2} & \{\text { similarly }\} .
\end{array}
$$

Therefore $P=Q_{1}=Q_{2}$. Since $P$ cannot be expressed as a linear combination of two distinct points, $P$ is a corner point of $R$. Since $P$ contains non-integral values for $s_{n m}$, relaxation $R$ is not tight.

\subsection{Improved model (model 4)}

A new model is proposed; although it maintains the same structure as that of the initial model, it does so using tighter constraints. The primary insight about the structure is expressed in the second constraint mentioned here. A node $n$ serves its child $m$ if and only if it serves all the nodes downstream of $m$. Consider set $S_{2}$ defined by the following set of constraints:

$$
\begin{aligned}
& \sum_{m} s_{m n}=1, \quad n=1, \ldots, N N, \quad m \in A_{n} \cup\{n\}, \\
& s_{n m}=s_{n k}, \quad n=1, \ldots, N N, \quad m \in C_{n}, \quad k \in D_{m}, \\
& s_{n m} \in\{0,1\}, \quad n=1, \ldots, N N, \quad m \in D_{n} \cup\{n\} .
\end{aligned}
$$

Proposition 5 The linear relaxation of $S_{2}$ is tight.

Proof Let the linear relaxation of set $S_{2}$ be $R_{2}$. Instead of constraint (58), we will have the following constraint:

$$
0 \leq s_{n m} \leq 1, \quad n=1, \ldots, N N, \quad m \in D_{n} \cup\{n\} .
$$

We will show that $R_{2}$ is tight by showing that any point $P$ with a non-integer component can be expressed as a linear combination of two distinct points from $R_{2}$.

Consider a point $P \in R_{2}$ with $0<s_{n^{\prime} n^{\prime}}=t<1$ for some $n^{\prime}$. Let $n^{\prime}$ be the first such node in the path from root.

Claim 5.1 $s_{n n}=1, \quad n \in A_{n^{\prime}}$.

Proof $s_{n n}$ cannot be fractional since $n^{\prime}$ is the first such node from root by definition. Assume $s_{n n}=0$ for some $n \in A_{n^{\prime}}$.

Let $E_{n n^{\prime}}=\left(D_{n} \cup\{n\}\right) \cap\left(A_{n}^{\prime} \cup\left\{n^{\prime}\right\}\right)$.

$$
\begin{aligned}
& s_{n n}=0 \\
& \equiv \quad\left\{\text { using } \sum_{m} s_{m n}=1(56)\right\} \\
& \begin{array}{ccc}
\sum_{m} s_{m n} & =1, & m \in A_{n} \\
\sum_{m} s_{m n^{\prime}}=1, & m \in A_{n}^{\prime} \cup\left\{n^{\prime}\right\}
\end{array} \\
& \equiv \quad \begin{array}{c}
\{\text { splitting sum }\} \\
\sum_{m} s_{m n^{\prime}}+\sum_{k} s_{k n^{\prime}}=1, \quad m \in A_{n}, k \in E_{n n^{\prime}}
\end{array} \\
& \equiv \quad \begin{array}{ll} 
& \left\{\text { using } s_{n m}=s_{n k}(62)\right\} \\
& \sum_{m} s_{m n}+\sum_{k} s_{k n^{\prime}}=1, \quad m \in A_{n}, k \in E_{n n^{\prime}}
\end{array} \\
& \equiv \quad\left\{\text { using } \sum_{m} s_{m n}=1 \text { from above }\right\} \quad k \in E_{n n^{\prime}} \\
& \equiv \quad\{\text { simplifying }\} \\
& \sum_{k} s_{k n^{\prime}}=0, \quad k \in E_{n n^{\prime}} \\
& \equiv \quad \begin{array}{c}
\text { using } \left.0 \leq s_{n m} \leq 1(63)\right\} \\
s_{k n^{\prime}}=0,
\end{array} \quad k \in E_{n n^{\prime}} \\
& \Rightarrow \quad\left\{\text { since } n^{\prime} \in E_{n n^{\prime}}\right\} \\
& s_{n^{\prime} n^{\prime}}=0 .
\end{aligned}
$$

However, this is a contradiction since we know that $s_{n^{\prime} n^{\prime}}$ is fractional. Therefore, $s_{n n}$ cannot be fractional and it cannot be 0 .

$$
s_{n n}=1, \quad n \in A_{n^{\prime}} .
$$

Claim 5.2 $s_{n m}=0, \quad n \in A_{p^{\prime}}, \quad m \in D_{n}, \quad p^{\prime}=P_{n^{\prime}}$. 


\section{Proof}

$$
\begin{aligned}
& s_{m m}=1, \quad m \in A_{n^{\prime}} \\
\equiv & \left\{\text { using } \sum_{m} s_{m n}=1(56)\right\} \\
& s_{n m}=0, \quad n \in A_{p^{\prime}}, \quad m \in A_{n^{\prime}}, \quad j \in D_{n}, \quad p^{\prime}=P_{n^{\prime}} \\
\equiv & \left\{\text { using } s_{n m}=s_{n k}(62)\right\} \\
& s_{n m}=0, \quad n \in A_{p^{\prime}}, \quad m \in D_{i}, \quad p^{\prime}=P_{n^{\prime}} .
\end{aligned}
$$

Consider a point $Q_{1}$ with $s_{n^{\prime} n^{\prime}}=0$ :

$$
\begin{gathered}
s_{n m}=s_{n m}^{P}, \quad m \notin\left(A_{n^{\prime}} \cup D_{n^{\prime}} \cup\left\{n^{\prime}\right\}\right) \\
s_{n m}=\frac{s_{n m}^{P}}{1-t}, \quad n \in A_{n^{\prime}}, j \in D_{n} \\
s_{n m}=0, \quad n \in\left(D_{n^{\prime}} \cup\left\{n^{\prime}\right\}\right), m \in D_{n} .
\end{gathered}
$$

\section{Claim 5.3 Point $Q_{1} \in R_{2}$.}

Proof We prove that point $Q_{1}$ belongs to $R_{2}$ by showing that it satisfies the constraints (56), (62) and (63).

For nodes that are not downstream or upstream of $n^{\prime}, s_{n m}$ values are the same as that of point $P$. Therefore, they satisfy the constraints since $P$ belongs to $R_{2}$.

For the rest of the nodes:

for $n \in A_{n^{\prime}}$ :

$$
\begin{gathered}
\operatorname{proving}(56): \quad \sum_{m} s_{m n}=1 \\
\left\{\operatorname{using} s_{n n}=1(64)\right\} \\
s_{n n}=1, \quad n \in A_{n^{\prime}} \\
\left\{\text { using } s_{n m}=0(65)\right\} \\
s_{m n}=0, \quad n \in A_{n^{\prime}}, \quad m \in A_{n} \\
\equiv \quad\{\text { summing over } m\} \\
\sum_{m} s_{m n}=1, \quad n \in A_{n^{\prime}} .
\end{gathered}
$$

Hence satisfied.

Proving (62): $s_{n m}=s_{n k}$

$$
\left\{\text { using } s_{n m}=s_{n k}(62)\right\}
$$$$
s_{n m}^{P}=s_{n k}^{P}, \quad n \in A_{n^{\prime}}, \quad m \in C_{n}, \quad k \in D_{m}
$$

$\equiv\{$ dividing by $(1-t)$ since $t \neq 1\}$

$$
\frac{s_{n m}^{P}}{1-t}=\frac{s_{n k}^{P}}{1-t}, \quad n \in A_{n^{\prime}}, \quad m \in C_{n}, \quad k \in D_{m}
$$$$
\equiv \quad\left\{\operatorname{using} s_{n m}=\frac{s_{n m}^{P}}{1-t}(67)\right\}
$$$$
s_{n m}=s_{n k}, \quad n \in A_{n^{\prime}}, \quad m \in C_{n}, \quad k \in D_{m} .
$$

Hence satisfied.

Proving (63): $\quad 0 \leq s_{m n^{\prime}} \leq 1$

$$
\begin{aligned}
& \left\{\text { using } \sum_{m} s_{m n}=1(56)\right\} \\
& \sum_{m} s_{m n^{\prime}}^{P}=1, \quad m \in A_{n^{\prime}} \cup\left\{n^{\prime}\right\} \\
\equiv & \{\text { splitting sum }\} \\
& \sum_{m} s_{m n^{\prime}}^{P}+s_{n^{\prime} n^{\prime}}^{P}=1, \quad m \in A_{n^{\prime}} \\
\equiv & \left\{\text { using } s_{n^{\prime} n^{\prime}}^{P}=t\right\} \\
& \sum_{m} s_{m n^{\prime}}^{P}=1-t, \quad m \in A_{n^{\prime}} \\
\equiv & \left\{\text { using } s_{m n^{\prime}}^{P} \geq 0(63)\right\} \\
& 0 \leq s_{m n^{\prime}}^{P} \leq 1-t, \quad m \in A_{n^{\prime}} \\
\equiv & \{\text { dividing by }(1-t) \text { since } t \neq 1\} \\
& 0 \leq \frac{s_{m n^{\prime}}^{P} \leq 1, \quad m \in A_{n^{\prime}}}{1-t} \leq 1, \quad s_{n m}^{P} \\
\equiv & \left\{\text { using } s_{n m}=\frac{s_{n-t}}{1-t}(67)\right\} \\
& 0 \leq s_{m n^{\prime}} \leq 1, \quad m \in A_{n^{\prime}} .
\end{aligned}
$$

Hence satisfied.

For $n \in D_{n^{\prime}} \cup\left\{n^{\prime}\right\}$, proving (56):

$$
\begin{aligned}
& \sum_{m} s_{m n}=1 \\
& \left\{\text { using } \sum_{m} s_{m n}=1(56)\right\} \\
& \sum_{m} s_{m n^{\prime}}^{P}=1, \quad m \in A_{n^{\prime}} \cup\left\{m^{\prime}\right\} \\
& \left\{\text { using } s_{n m}=0(65)\right\} \\
& s_{k n^{\prime}}^{P}+s_{n^{\prime} n^{\prime}}^{P}=1, \quad k=P_{n^{\prime}} \\
\equiv & \left\{\text { using } s_{n^{\prime} n^{\prime}}=t\right\} \\
& s_{k n^{\prime}}^{P}=1-t, \quad k=P_{n^{\prime}} \\
\equiv & \left\{\text { using } s_{n m}=\frac{s_{n m}^{P}}{1-t}(67)\right\} \\
& s_{k n^{\prime}}=1, \quad k=P_{n^{\prime}} \\
\equiv & \left\{\text { using } s_{n m}=0(68)\right\} \\
& \sum_{m} s_{m n}=1, \quad n \in D_{n^{\prime}} \cup\left\{n^{\prime}\right\}, m \in A_{n} .
\end{aligned}
$$

Hence satisfied.

Proving (62) :

$$
\begin{aligned}
& \quad s_{n m}=s_{n k} \\
& \quad\left\{\text { using } s_{n m}=0(68)\right\} \\
& s_{n m}=0, \quad n \in D_{n^{\prime}} \cup\left\{n^{\prime}\right\}, m \in D_{n} \\
& \equiv \\
& s_{n m}=s_{n k}, \quad n \in D_{n^{\prime}} \cup\left\{n^{\prime}\right\}, m \in D_{n}, k \in C_{n} .
\end{aligned}
$$


Hence satisfied.

Proving (63) :

$$
\begin{aligned}
& 0 \leq s_{n m} \leq 1 \\
& \quad\left\{\text { using } s_{n m}=0(68)\right\} \\
& s_{n m}=0, \quad n \in D_{n^{\prime}} \cup\left\{n^{\prime}\right\}, m \in D_{n} .
\end{aligned}
$$

Hence satisfied.

Therefore point $Q_{1} \in R_{2}$.

Similarly, consider point $Q_{2}$ with $s_{n^{\prime} n^{\prime}}=1$ :

$$
\begin{gathered}
s_{n m}=s_{n m}^{P}, \quad m \notin\left(A_{n^{\prime}} \cup D_{n^{\prime}} \cup\left\{n^{\prime}\right\}\right) \\
s_{n m}=0, \quad n \in A_{n^{\prime}}, m \in D_{n} \\
s_{n m}=\frac{s_{n m}^{P}}{t}, \quad n \in\left(D_{n^{\prime}} \cup\left\{n^{\prime}\right\}\right), m \in D_{n} \cup\{n\} .
\end{gathered}
$$

Claim 5.4 Point $Q_{2} \in R_{2}$.

Using a similar argument as before, we can prove that point $Q_{2}$ belongs to $R_{2}$ by showing that it satisfies the constraints (56), (62) and (63).

Claim 5.5 $P$ is a linear combination of points $Q_{1}$ and $Q_{2}$, i.e. $P=(1-t) Q_{1}+t Q_{2}$.

Proof For $m \notin\left(A_{n^{\prime}} \cup D_{n^{\prime}} \cup\left\{n^{\prime}\right\}\right)$ :

$$
\begin{aligned}
& \quad\left\{\text { using } s_{n m}^{P}=s_{n m}^{Q_{1}}(66) \text { and } s_{n m}^{P}=s_{n m}^{Q_{2}}(69)\right\} \\
& s_{n m}^{P}=s_{n m}^{Q_{1}}=s_{n m}^{Q_{2}} \\
\Rightarrow & s_{n m}^{P}=(1-t) s_{n m}^{Q_{1}}+t s_{n m}^{Q_{2}} .
\end{aligned}
$$

For $n \in A_{n^{\prime}}, m \in D_{n}$ :

$$
\begin{aligned}
s_{n m}^{Q_{1}} & =\frac{s_{n m}^{P}}{1-t} \\
s_{n m}^{Q_{2}} & =0 \\
\Rightarrow & s_{n m}^{P}=(1-t) s_{n m}^{Q_{1}}+t s_{n m}^{Q_{2}} .
\end{aligned}
$$

For $n \in D_{n^{\prime}}, m \in D_{n}$ :

$$
\begin{aligned}
s_{n m}^{Q_{1}} & =0 \\
s_{n m}^{Q_{2}} & =\frac{s_{n m}^{P}}{t} \\
\Rightarrow & s_{n m}^{P}=(1-t) s_{n m}^{Q_{1}}+t s_{n m}^{Q_{2}} .
\end{aligned}
$$

Therefore, $P$ is a linear combination of points $Q_{1}$ and $Q_{2}$.

Since any general point $P$ with a fractional component can be expressed as a linear combination of two other points in the set $R_{2}$, it implies that such a point $P$ cannot be a corner point and therefore set $R_{2}$ is tight.
This concludes the discussion on the three improvements made to the initial ILP model. Experimental results of the performance of the model after each improvement are presented in section 7. Although we have shown the tightness of various subsets of the improved model, the overall set of constraints of the model is still not tight. As such, there remains room for further improvements to the model. In the next section, we describe an initial attempt at an alternative approach to the problem. Instead of using node variables $s_{i j}$ to partition the primary and secondary network, variables based purely on edges are used.

\section{Edge-based model}

An alternative approach to the node-based representation of the network is to have an edge-based representation. Here, instead of the focus being which tank serves which node, the focus is on which pipes in the network are part of the primary network and which pipes are part of the secondary network. Consider a tree network of $N E$ edges.

\section{Parameters}

$C_{i}=$ set of pipes that are immediately downstream of pipe $i, \quad i=1, \ldots, N E$.

$U_{i}=$ set of pipes that are upstream of pipe

$i, \quad i=1, \ldots, N E$.

$D_{i}=$ set of pipes that are downstream of pipe $i, \quad i=1, \ldots, N E$.

$D S=$ set of pipes that are immediately downstream of the source.

Variables:

$f_{i}=1$ if $i^{\text {th }}$ edge belongs to the primary network and $=0$ if $i^{\text {th }}$ edge belongs to the secondary network, $i=1, \ldots, e$.

The primary network connects the source to the tanks, and the secondary network connects the tanks to downstream nodes. Therefore, pipes starting from the source must belong to the primary network. Also, secondary pipes must be downstream of the primary pipes. Once a pipe is secondary, any pipes downstream can no longer be primary. We can use the following set of constraints to describe the set $S_{3}$ of valid network configurations:

$$
\begin{gathered}
f_{i}=1, \quad i \in D S, \\
f_{j} \leq f_{i}, \quad i=1, \ldots, N E, \quad j \in C_{i}, \\
f_{i} \in\{0,1\}, \quad i=1, \ldots, N E .
\end{gathered}
$$

Proposition 6 The linear relaxation of $S_{3}$ is tight.

Proof Let the relaxation of set $S_{3}$ be $R_{3}$. Instead of constraint (74), we will have the following constraint:

$$
0 \leq f_{i} \leq 1, \quad i=1, \ldots, N E
$$


We will show that $R_{3}$ is tight by showing that any point $P$ with a non-integer component can be expressed as a combination of two distinct points from $R_{3}$.

Consider a point $P \in R_{3}$ with $0<f_{i}=t<1$ for some $i^{\prime}$. Let $i^{\prime}$ be the first such edge in the path from source.

Claim 6.1 $f_{i}=1, \quad i \in U_{i^{\prime}}$.

Proof $f_{i}$ cannot be fractional since $i^{\prime}$ is the first such edge from the source by definition. If $f_{i}=0$, then by (73) for all its downstream edges $j, f_{i}=0$. However, $i^{\prime}$ is downstream of $i$ and $f_{i^{\prime}}=t \neq 0$. Therefore, $f_{i}$ cannot be fractional and it cannot be 0 .

$$
f_{i}=1, \quad i \in U_{i^{\prime}} .
$$

Consider a point $Q_{1}$ with $f_{i^{\prime}}=0$ :

$$
\begin{aligned}
& f_{i}=f_{i}^{P}, \quad i \notin\left(D_{i^{\prime}} \cup\left\{i^{\prime}\right\}\right) \\
& f_{i}=0, \quad i \in\left(D_{i^{\prime}} \cup\left\{i^{\prime}\right\}\right) .
\end{aligned}
$$

Claim 6.2 Point $Q_{1} \in R_{3}$.

Proof For all the edges not downstream of $i^{\prime}$, constraints (72), (73) and (75) are satisfied since the values are same as point $P$ and $P \in R_{3}$. Setting $f_{i}=0$ for all downstream $i$ also maintains the constraints trivially. Therefore point $Q \in R_{3}$.

Similarly consider point $Q_{2}$ with $f_{i^{\prime}}=1$ :

$$
\begin{aligned}
& f_{i}=f_{i}^{P}, \quad i \notin\left(D_{i^{\prime}} \cup\left\{i^{\prime}\right\}\right) \\
& f_{i}=\frac{f_{i}^{P}}{t}, \quad i \in\left(D_{i^{\prime}} \cup\left\{i^{\prime}\right\}\right)
\end{aligned}
$$

Claim 6.3 Point $Q_{2} \in R_{3}$.

Proof We prove that point $Q_{2}$ belongs to $R_{3}$ by showing it satisfies the constraints (72), (73) and (75). For edges that are not downstream of $i^{\prime}, f_{i}$ values are same as that of point $P$. Therefore they satisfy the constraints since $P \in R_{3}$. For the rest of the edges:

For $i \in\left(D_{i^{\prime}} \cup\left\{i^{\prime}\right\}\right)$ : (72) is trivially true since $i^{\prime}$ (and its downstream edges) cannot be connected to the source since for point $P, f_{i} \neq 0$.

$$
\begin{gathered}
\text { Proving (73): } f_{j} \leq f_{i} \\
\left\{\operatorname{using} f_{j}^{P} \leq f_{i}^{P}(73)\right\} \\
f_{j}^{P} \leq f_{i}^{P}, \quad i \in\left(D_{i^{\prime}} \cup\left\{i^{\prime}\right\}\right), j \in C_{i} \\
\equiv \quad\{\text { dividing by } t \text { since } t \neq 0\} \\
\quad \frac{f_{j}^{P}}{t} \leq \frac{f_{i}^{P}}{t}, \quad i \in\left(D_{i^{\prime}} \cup\left\{i^{\prime}\right\}\right), j \in C_{i} \\
\equiv \quad\left\{\operatorname{using} f_{i}=\frac{f_{i}^{P}}{t}(80)\right\}
\end{gathered}
$$

$$
\begin{aligned}
& f_{j} \leq f_{i}, \quad i \in\left(D_{i^{\prime}} \cup\left\{i^{\prime}\right\}\right), j \in C_{i} . \\
& \text { Hence satisfied. } \\
& \text { Proving }(75): \quad 0 \leq f_{i} \leq 1 \\
& \quad\left\{\operatorname{using} f_{i}^{P} \leq f_{i^{\prime}}^{P}(73) \text { and } 0 \leq f_{i}^{P}(75)\right\} \\
& 0 \leq f_{i}^{P} \leq f_{i^{\prime}}^{P} \quad i \in\left(D_{i^{\prime}} \cup\left\{i^{\prime}\right\}\right) \\
& \equiv \quad\left\{\text { using } f_{i}^{P}=t\right\} \\
& 0 \leq f_{i}^{P} \leq t \quad i \in\left(D_{i^{\prime}} \cup\left\{i^{\prime}\right\}\right) \\
& \equiv \quad\{\operatorname{dividing} \text { by } t \text { since } t \neq 0\} \\
& \left.0 \leq \frac{f_{i}^{P} \leq 1 \quad i \in\left(D_{i^{\prime}} \cup\left\{i^{\prime}\right\}\right)}{t} \leq 1 \quad \text { using } f_{i}=\frac{f_{i}^{P}}{t}(80)\right\} \\
& \equiv \leq f_{i} \leq 1 \quad i \in\left(D_{i^{\prime}} \cup\left\{i^{\prime}\right\}\right) .
\end{aligned}
$$

Hence satisfied.

Therefore point $Q_{2} \in R_{3}$.

Claim 6.4 $P$ is a linear combination of points $Q_{1}$ and $Q_{2}$ i.e. $P=(1-t) Q_{1}+t Q_{2}$

Proof For $i \notin\left(D_{i^{\prime}} \cup\left\{i^{\prime}\right\}\right)$ :

$$
\begin{gathered}
f_{i}^{P}=f_{i}^{Q_{1}}=f_{i}^{Q_{2}} \\
\Rightarrow \quad f_{i}^{P}=(1-t) f_{i}^{Q_{1}}+t f_{i}^{Q_{2}}
\end{gathered}
$$

For $i \in\left(D_{i^{\prime}} \cup\left\{i^{\prime}\right\}\right)$ :

$$
\begin{gathered}
f_{i}^{Q_{1}}=0 \\
f_{i}^{Q_{2}}=\frac{f_{i}^{P}}{t} \\
\Rightarrow \quad f_{i}^{P}=(1-t) f_{i}^{Q_{1}}+t f_{i}^{Q_{2}} .
\end{gathered}
$$

Therefore $P$ is a linear combination of points $Q_{1}$ and $Q_{2}$

Since any general point $P$ with a fractional component can be expressed as a linear combination of two other points in the set $R_{3}$, it implies that such a point $P$ cannot be a corner point and therefore relaxation $R_{3}$ is tight.

The performance of the edge-based model is worse than that of model 4. Although we prove that the LP relaxation of the set of constraints described by $S_{3}$ is tight, the LP relaxation objective for the overall model is worse. This is due to changes in other constraints of the model, which are required since in this model only edge-based variables are considered.

\section{Computational results}

The three pipe cost/tank cost/tank allocation improvements were applied sequentially to the initial model (model 1) to give model $2 /$ model $3 /$ model 4 , respectively. These four 


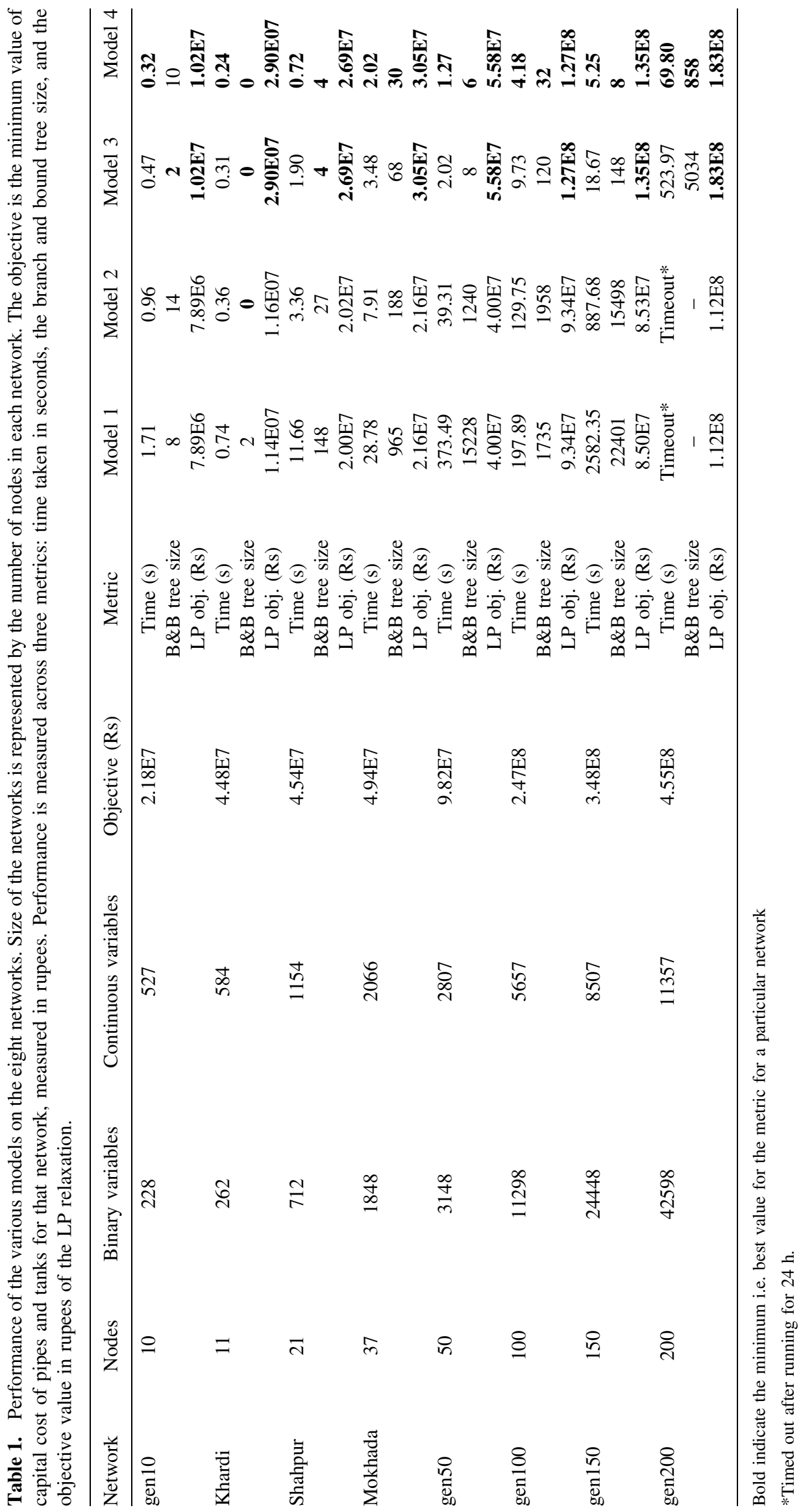


models were tested over eight different networks of varying sizes in order to test their performance and scalability.

- Real world networks: Three of the networks-Khardi, Shahpur and Mokhada-are real life networks from Maharashtra state in India. These regions consist of tribal villages that regularly face extreme water stress during summer months and as a result have to be provided water using tankers. Detailed information regarding the networks can be found at [13] and [14]. Ranges for the node and link properties are as follows.

- Number of children nodes: 1-3.

- Elevation (in metres): 202-470.

- Demand (in 1/second): 0.48-9.24.

- Length of links (in metres): 187-9911.

- Synthetic networks: The other five networks are artificially created to test the performance of the models across different network sizes (10-200). Each of them is a randomly generated branched network. Ranges for the node and link properties are as follows.

- Number of children nodes: 1-5.

- Elevation (in metres): 100-300.

- Demand (in litres per second): 0.01-5.

- Length of links (in metres): 500-5000.

For all four models, the problem statement remains the same - to optimize the total pipe and tank cost of the network. The numbers of binary and continuous variables scale with the size of the network. Since all four models solve the problem optimally, the final capital cost of the pipes and tanks is the same. The performance of each model is measured in terms of three metrics: the total time taken in seconds, the size of the branch and bound tree and the objective value of the LP relaxation. The tests were run on an Intel Core i5-4210U @ 1.7-GHz machine with 4-GB RAM and running a 64-bit Windows 8.1 operating system. Table 1 summarizes the performance of the models. We observe that for each of the eight networks, the time taken improves with each model, resulting in model 4 providing the best performance. Typically, the time taken scales with the size of the network. However, this need not always be the case. For example, although gen50 has more nodes (50 nodes) than Mokhada (37 nodes), it is solved in lesser amount of time. This is because apart from the number of nodes being a factor, the network configuration also matters while solving the model.

\section{Conclusion}

In the present work we looked at the cost optimization of rural drinking water schemes. These schemes consist of several network components like pipes, tanks, pumps and valves. We first describe an initial ILP model that was used to solve the optimization. Although optimal, the model took a significant amount of time for larger networks-45 min for a network with 150 nodes. We then describe a series of three improvements of the model. For each improvement, we prove that the improved model is tighter than the initial model. We then finally present the performance results of the three improved models along with the initial model over eight networks of various sizes. The 150-node network now takes only $5 \mathrm{~s}$ to solve.

Thus, we show that tightening the ILP model can result in significant improvements in terms of performance. This enables practitioners to consider greater number of iterations of the design for large networks, since each iteration can be optimized in a matter of seconds. In addition, although improvements were made in several constraints, the overall model is still not tight. Further improvements are possible to increase performance even further. Additionally, the model can be extended in the future to include looped networks and/or consider scheduling of the water distribution.

\section{References}

[1] Alperovits E and Shamir U 1977 Design of optimal water distribution systems. Water Resources Research 13(6): 885-900

[2] Liang T, Yang K P and Wu I P 1974 Dynamic programming optimization: a water distribution system design. Water Resources Research Center, University of Hawaii

[3] Lansey K E and Mays L W 1989 Optimization model for water distribution system design. Journal of Hydraulic Engineering 115(10): 1401-1418.

[4] Samani H M and Mottaghi A 2006 Optimization of water distribution networks using integer linear programming. Journal of Hydraulic Engineering, 132(5): 501-509

[5] Yates D F, Templeman A B and Boffey T B 1984 The computational complexity of the problem of determining least capital cost designs for water supply networks. Engineering Optimization 7(2): 143-155

[6] Savic D A and Walters G A 1997 Genetic algorithms for least-cost design of water distribution networks. Journal of Water Resources Planning and Management 123(2): 67-77

[7] Nicklow J, Reed P, Savic D, Dessalegne T, Harrell L, ChanHilton A ... and Zechman E 2009 State of the art for genetic algorithms and beyond in water resources planning and management. Journal of Water Resources Planning and Management 136(4): 412-432

[8] da Conceicao Cunha M and Ribeiro L 2004 Tabu search algorithms for water network optimization. European Journal of Operational Research 157(3): 746-758

[9] Eusuff M M and Lansey K E 2003 Optimization of water distribution network design using the shuffled frog leaping algorithm. Journal of Water Resources Planning and Management 129(3): 210-225

[10] Swamee P K, Kumar V and Khanna P 1973 Optimization of dead end water distribution systems. Journal of the Environmental Engineering Division 99(2): 123-134 
[11] WaterGEMS, Bentley Systems 2006 Bentley Systems Incorporated, Exton, PA

[12] Modak P M and Dhoonia J 1991 A computer program in Quick BASIC for the least cost design of branched water distribution networks. Asia Water Supply and Sanitation Sector Development Project, UNDP/World Bank, RAS/86/160

[13] Hooda N, Desai R and Damani O P 2013 Design and optimization of piped water network for tanker fed villages in Mokhada Taluka. Technical Report No. TR-CSE-2013e-55, Department of Computer Science and Engineering, IIT Bombay

[14] Choudhary V, Damani O, Desai R, Joshi A, Kanwat M, Kaushal M et al 2013 Redesigning Khardi rural piped water network scheme for sustainability. Technical Report No. TRCSE-2013-56, Department of Computer Science and Engineering, IIT Bombay
[15] Vyas J H, Shrimali N J and Modi M A 2014 Optimization of Dhrafad Regional Water Supply Scheme using Branch 3.0. International Journal of Innovative Research in Science, Engineering and Technology

[16] Lad Y, Main J S and Chawathe S D 2012 Optimization of hydraulic design of water supply tree network based on present worth analysis. Journal of Indian Water Works Association (January-March): 67-71

[17] Hooda N and Damani O 2017 A system for optimal design of pressure constrained branched piped water networks. Procedia Engineering 186: 349-356

[18] Hooda N and Damani O 2017 Inclusion of tank configurations as a variable in the cost optimization of branched piped-water networks. Drinking Water Engineering and Science 10(1): 39-44

[19] Williams G S and Hazen A 1908 Hydraulic tables.. J. Wiley \& Sons, London 